\title{
The Relation between Relativistic Strings and Maxwell Fields of Rank 2
}

\section{Max Rinke}

Institut für Theoretische Physik, RWTH Aachen, D-5100 Aachen, Federal Republic of Germany

\begin{abstract}
The local correspondence between sufficiently smooth relativistic string motions and Maxwell fields of rank 2 is proved.
\end{abstract}

In two recent papers [1,2] Kastrup studied an electromagnetic field which is connected in a special way with a relativistic string $[3,4]$.

He started from the following string motion $x(\tau, \sigma)$ :

$$
\begin{array}{ll}
x^{0}=\tau, & x^{1}=A\left(\sigma-\frac{\pi}{2}\right) \cos \omega \tau, \quad x^{2}=A\left(\sigma-\frac{\pi}{2}\right) \sin \omega \tau, \\
x^{3}=0, \quad 0 \leqq \sigma \leqq \pi, \quad A \omega=\frac{2}{\pi},
\end{array}
$$

which may be interpreted geometrically as a 2-dimensional surface $\Sigma^{(2)}$ in Minkowski space. Then Kastrup introduced Plücker's coordinates on $\Sigma^{(2)}$ :

$$
v^{\mu \nu}:=\dot{x}^{\mu} x^{\prime \nu}-\dot{x}^{v} x^{\prime \mu}, \quad \dot{x}^{\mu}:=\frac{\partial x^{\mu}}{\partial \tau}, \quad x^{\prime \mu}:=\frac{\partial x^{\mu}}{\partial \sigma},
$$

and constructed an electromagnetic field $F^{\mu v}(x)$ which is proportional on $\Sigma^{(2)}$ to $v^{\mu \nu}$ :

$$
F^{\mu v}(x(\tau, \sigma))=\lambda v^{\mu v}(\tau, \sigma), \quad \lambda=\text { const } .
$$

The nontrivial part of the problem is to ensure that the field $F^{\mu v}(x)$ is a solution of the homogeneous Maxwell's equations :

$$
\partial_{\mu}^{*} F^{\mu \nu}=0, \quad{ }^{*} F_{\mu \nu}=\frac{1}{2} \varepsilon_{\mu \nu \varrho \sigma} F^{\varrho \sigma}, \quad \partial_{\mu}=\frac{\partial}{\partial x^{\mu}} .
$$

[We use the metric $(+1,-1,-1,-1) ; \hbar=c=1$.]

The remaining (inhomogeneous) Maxwell's equations may be taken as a definition of the current $j^{v}:=\partial_{\mu} F^{\mu v}$ ! 
The field $F^{\mu v}$, which Kastrup constructed, has the following form:

$$
\begin{aligned}
F^{01} & =\lambda A \cos \omega t, & F^{02} & =\lambda A \sin \omega t, \quad F^{03}=0, \\
F^{12} & =-\lambda A \omega \varrho, & F^{13} & =F^{23}=0 ; \\
t & =x^{0}, & \varrho & :=\left[\left(x^{1}\right)^{2}+\left(x^{2}\right)^{2}\right]^{1 / 2} .
\end{aligned}
$$

It is a field of rank 2, i.e.

$$
{ }^{*} F_{\mu \nu} F^{\mu v}=4 \mathbf{E} \cdot \mathbf{B}=0,
$$

and satisfies condition (3) for $\frac{\pi}{2} \leqq \sigma \leqq \pi$.

The existence of the Maxwell field (4) is not accidental. We want to show that there is an intimate relation between Maxwell fields of rank 2 and sufficiently smooth solutions of the equations of motion for the relativistic string. Our result is contained in the following two theorems:

Theorem 1. Let the 2-dimensional surface $\Sigma^{(2)}$ with the parametrization $x(\tau, \sigma)$ and Plücker's coordinates $v^{\mu v}(\tau, \sigma)$ represent a four times continuously differentiable string motion, defined by the Lagrangian density $L=\left(-\frac{1}{2} v_{\mu \nu} v^{\mu \nu}\right)^{1 / 2}$. Let $x_{(0)}=x\left(\tau_{0}, \sigma_{0}\right)$ be a point on $\Sigma^{(2)}$, such that Plücker's coordinates $v_{(0)}^{\mu v}=v^{\mu v}\left(\tau_{0}, \sigma_{0}\right)$ satisfy the condition

$$
-\frac{1}{2} v_{(0)}^{\mu v} v_{(0) \mu \nu}>0 \text {. }
$$

Then, for arbitrary fixed $\lambda \in \mathbb{R}, \lambda \neq 0$, there exists a neighborhood $U_{0}$ of $x_{(0)}$ in Minkowski space and a continuously differentiable Maxwell field $F^{\mu \nu}(x)$ of rank 2, defined in $U_{0}$, such that

$$
F^{\mu v}(x(\tau, \sigma))=\lambda v^{\mu v}(\tau, \sigma), \quad \lambda=\text { const }, \quad x(\tau, \sigma) \in U_{0},
$$

and

$$
-\frac{1}{2} F^{\varrho \sigma}(x) F_{\varrho \sigma}(x)>0, \quad \partial_{\mu}\left[{ }^{*} F^{\mu v}(x)\left(-\frac{1}{2} F^{\varrho \sigma}(x) F_{\varrho \sigma}(x)\right)^{-1 / 2}\right]=0 .
$$

Theorem 2. Let $F^{\mu v}(x)$ be a continuously differentiable Maxwell field of rank 2 with

$$
-\frac{1}{2} F^{\varrho \sigma}(x) F_{\varrho \sigma}(x)>0, \quad \partial_{\mu}\left[F^{\mu v}(x)\left(-\frac{1}{2} F^{\varrho \sigma}(x) F_{\varrho \sigma}(x)\right)^{-1 / 2}\right]=0 .
$$

Let $\Sigma^{(2)}$ be a 2-dimensional surface with twice continuously differentiable parametrization $x(\tau, \sigma)$ and Plücker's coordinates $v^{\mu \nu}(\tau, \sigma)$, such that the relation

$$
F^{\mu v}(x(\tau, \sigma))=\lambda v^{\mu v}(\tau, \sigma), \quad \lambda=\text { const },
$$

is valid on $\Sigma^{(2)}$. Then $x(\tau, \sigma)$ is a solution of the equations of motion for the relativistic string.

For the proof of these theorems we need some definitions and results from the calculus of variations in the formulation of Carathéodory [5]. Here we refer especially to the work of Velte [6,7] and Klötzler [8].

We start from the Lagrangian density $L$ for the relativistic string

$$
L(v):=\left[-\frac{1}{2} v_{\mu \nu} v^{\mu \nu}\right]^{1 / 2}=\left[-v_{(\mu \nu)} v^{(\mu \nu)}\right]^{1 / 2},
$$

normalizing any constant factor to 1 . 

$\mu<v$.

By $v^{(\mu v)}$ and $v_{(\mu v)}$ we denote the "strict components" of $v$, i.e. $v^{\mu v}$ resp. $v_{\mu \nu}$ with

The action principle, applied to the string action $A=\int L d \tau d \sigma$, leads to the equations of motion:

$$
\frac{\partial}{\partial \tau}\left(\frac{\partial L}{\partial \dot{x}^{\mu}}\right)+\frac{\partial}{\partial \sigma}\left(\frac{\partial L}{\partial x^{\prime \mu}}\right)=0 .
$$

$L(v)$ is a homogeneous function of degree 1 :

$$
L(\kappa v)=\kappa L(v), \quad \kappa>0 .
$$

Therefore, the action of the string is invariant under reparametrizations, and, using the definition

$$
L_{(\mu v)}(v):=\frac{\partial L(v)}{\partial v^{(\mu v)}}=-\frac{v_{(\mu v)}}{L(v)},
$$

we obtain :

$$
L_{(\mu v)}(v) v^{(\mu v)}=L(v)
$$

In order to have well defined derivatives $\frac{\partial L}{\partial \dot{x}^{\mu}}, \frac{\partial L}{\partial x^{\prime \mu}}, L_{(\mu v)}(v)$ and for reasons to be seen later, we assume

$$
L(v)>0 \text {. }
$$

The usual boundary conditions for the string

$$
\frac{\partial L}{\partial x^{\prime \mu}}=0, \quad \sigma=0, \pi
$$

lead to $L(v)=0$ for $\sigma=0, \pi$. This shows that $L(v)>0$ cannot be valid for all $\tau, \sigma$. So we restrict our considerations to such values of $\tau, \sigma$, for which the condition (11) is fulfilled.

From the definition (2) of Plücker's coordinates $v^{\mu v}$ we find the characteristic condition

$$
v^{\mu \nu} \varepsilon_{\mu \nu \varrho \sigma} v^{\varrho \sigma}=4 v^{(\mu \nu)} \varepsilon_{\mu \nu \varrho \sigma} v^{(\varrho \sigma)}=0 .
$$

Assume now that at every point $x$ in a region of Minkowski space a 2-dimensional surface element, i.e. a 2-dimensional tangent space at $x$, is given, characterized by Plücker's coordinates $\Phi^{\mu v}(x)$ :

$$
\Phi^{\mu v}=-\Phi^{v \mu}, \quad \Phi^{\mu v} \varepsilon_{\mu \nu \varrho \sigma} \Phi^{\varrho \sigma}=4 \Phi^{(\mu v)} \varepsilon_{\mu \nu \varrho \sigma} \Phi^{(\varrho \sigma)}=0 .
$$

We call $\left(x, \Phi^{\mu v}(x)\right)=(x, \Phi(x))$ a "slope field" of 2-dimensional surface elements.

In the following we make the additional assumption that the functions $\Phi^{\mu v}(x)$ are continuously differentiable. 
Definition. The slope field $(x, \Phi(x))$ is called "geodesic with respect to the Lagrangian density $L$ " if the exterior differential form

$$
\Omega:=L_{(\mu v)}(\Phi(x)) d x^{\mu} \wedge d x^{v}
$$

is closed, i.e. $d \Omega=0$.

Let $S_{1}(x), S_{2}(x)$ be twice continuously differentiable functions. We then define:

$$
S_{\mu \nu}(x):=\frac{\partial S_{1}(x)}{\partial x^{\mu}} \frac{\partial S_{2}(x)}{\partial x^{\nu}}-\frac{\partial S_{1}(x)}{\partial x^{\nu}} \frac{\partial S_{2}(x)}{\partial x^{\mu}} .
$$

Again, we denote the corresponding strict components by $s_{(\mu \nu)}(x)$. The differential form

$$
d S_{1} \wedge d S_{2}=S_{(\mu \nu)}(x) d x^{\mu} \wedge d x^{v}
$$

is exact. Thus, $\Omega$ is certainly a closed form if

$$
\Omega=d S_{1} \wedge d S_{2}, \text { i.e. } \quad L_{(\mu v)}(\Phi(x))=S_{(\mu \nu)}(x) .
$$

On the other hand, according to Eqs. (10) and (12), we have

$$
L_{(\mu \nu)}(\Phi) \varepsilon^{\mu \nu \varrho \sigma} L_{(\varrho \sigma)}(\Phi)=0 .
$$

This shows that the closed 2-form $\Omega$ has rank 2 . Therefore, $\Omega$ can locally be represented in the form (14), [9], and we have the result: $(x, \Phi(x))$ is a geodesic field with respect to $L$ if and only if $\Omega$ locally has a representation of the form (14).

Definition. A surface $\Sigma^{(2)}$ with parametrization $x(\tau, \sigma)$ and Plücker's coordinates $v^{\mu v}(\tau, \sigma)$ is called "imbedded" in the geodesic field $(x, \Phi(x))$ if there exists a function $\gamma(\tau, \sigma)>0$ with

$$
v^{\mu \nu}(\tau, \sigma)=\gamma(\tau, \sigma) \Phi^{\mu v}(x(\tau, \sigma)) .
$$

Then $(x, \Phi(x))$ is called a "geodesic field for $x(\tau, \sigma)$ ".

In the calculus of variations it is shown [6-8] that for any sufficiently smooth solution of the equations of motion (9) a geodesic field can be constructed in the following way:

One starts from the representation (14) of $\Omega$. From this representation and the Eqs. (8), (10) it follows that the functions $S_{1}(x), S_{2}(x)$ have to satisfy the so-called "generalized Hamilton-Jacobi differential equation" for the string problem:

$$
\left[-s_{(\mu \nu)}(x) s^{(\mu \nu)}(x)\right]^{1 / 2}=1 .
$$

Let $x(\tau, \sigma)$ be a string solution which shall be imbedded in a geodesic field. Then one looks for solutions $S_{1}(x), S_{2}(x)$ of Eq. (15), which in addition fulfill the following "transversality condition":

$$
s_{(\mu v)}(x(\tau, \sigma))=L_{(\mu \nu)}(v(\tau, \sigma)) .
$$

Let $S_{1}(x), S_{2}(x)$ be such solutions. Then $(x, \Phi(x))$ with

$$
\Phi_{\mu \nu}(x):=-s_{\mu \nu}(x)
$$


is a geodesic field for $x(\tau, \sigma)$ :

$$
\begin{aligned}
L_{(\mu v)}(\Phi(x)) & =-\frac{\Phi_{(\mu v)}(x)}{L(\Phi)}=\frac{S_{(\mu v)}(x)}{L(-s)}=s_{(\mu v)}(x), \\
v_{(\mu v)} & =-L(v) L_{(\mu v)}(v)=-L(v) s_{(\mu v)}(x(\tau, \sigma))=L(v) \Phi_{(\mu v)}(x(\tau, \sigma)) .
\end{aligned}
$$

Conversely, if $(x, \Phi(x))$ is a geodesic field for $x(\tau, \sigma)$ with

$$
L_{(\mu \nu)}(\Phi)=S_{(\mu v)}, \quad S_{\mu \nu}=\partial_{\mu} S_{1} \partial_{v} S_{2}-\partial_{\nu} S_{1} \partial_{\mu} S_{2},
$$

then $S_{1}(x), S_{2}(x)$ satisfy the Eqs. (15) and (16).

In $[6,7]$ it is proved that simultaneous solutions of Eqs. (15), (16) exist if $x(\tau, \sigma)$ is sufficiently smooth. We state this result as Theorem A.

Theorem A. Let $x(\tau, \sigma)$ be a four times continuously differentiable solution of the equations of motion (9) with Plücker's coordinates $v^{\mu v}$ and $-\frac{1}{2} v^{\mu v} v_{\mu v}>0$. Then there exists always locally a geodesic field for $x(\tau, \sigma)$.

We now come to the Proof of Theorem 1:

From Theorem A we conclude that for $x_{(0)}=x\left(\tau_{0}, \sigma_{0}\right)$ with $-\frac{1}{2} v_{(0)}^{\mu v} v_{(0) \mu \nu}>0$ we can find a neighborhood $U_{1}$ in Minkowski space and twice continuously differentiable functions $S_{1}(x), S_{2}(x)$, which are defined in $U_{1}$ and satisfy the Eqs. (15) and (16).

If $L(v(\tau, \sigma))=\kappa=$ const for $x(\tau, \sigma) \in U_{1}$, then for a given $\lambda \neq 0$

$$
F_{\mu \nu}(x):=-\lambda \kappa s_{\mu \nu}(x), \quad x \in U_{0}:=U_{1},
$$

is a Maxwell field of rank 2, which fulfills the conditions (5) and (6).

If $L(v)$ is not constant in $U_{1}$, we introduce new parameters $\tau^{\prime}, \sigma^{\prime}$ :

$$
\tau^{\prime}:=S_{1}(x(\tau, \sigma)), \quad \sigma^{\prime}:=S_{2}(x(\tau, \sigma)) .
$$

The corresponding Jacobian determinant $D$ has the following form:

$$
D=\frac{\partial\left(\tau^{\prime}, \sigma^{\prime}\right)}{\partial(\tau, \sigma)}=s_{(\mu v)}(x(\tau, \sigma)) v^{(\mu v)}(\tau, \sigma)=L(v)
$$

Since $L\left(v_{(0)}\right)>0$, the transformation $(\tau, \sigma) \rightarrow\left(\tau^{\prime}, \sigma^{\prime}\right)$ has an inverse if we restrict ourself to an appropriate neighborhood of $\left(\tau_{0}, \sigma_{0}\right)$ :

$$
\tau=\tau\left(\tau^{\prime}, \sigma^{\prime}\right), \quad \sigma=\sigma\left(\tau^{\prime}, \sigma^{\prime}\right) .
$$

With the help of these equations we express $L(v)$ as a function of $\tau^{\prime}, \sigma^{\prime}$ :

$$
L(v)=: g\left(\tau^{\prime}, \sigma^{\prime}\right)
$$

Now, let $U_{0} \subseteq U_{1}$ be a neighborhood of $x_{(0)}$, such that $g\left(S_{1}(x), S_{2}(x)\right)$ is well defined and positive in $U_{0}$. Then for a given $\lambda \neq 0$ we define:

$$
F_{\mu \nu}(x):=-\lambda g\left(S_{1}(x), S_{2}(x)\right) s_{\mu v}(x), \quad x \in U_{0} .
$$

The special $x$-dependence of $g$ and the form (13) of $s_{\mu \nu}(x)$ imply that the field $F_{\mu \nu}$ is a rank-2 solution of Maxwell's equations (with an appropriate current). Finally, 
the Eqs. (15) and (16) ensure that the field $F_{\mu \nu}$ also satisfies the conditions (5) and (6).

Remark 1. By an appropriate reparametrization $(\tau, \sigma) \rightarrow\left(\tau^{\prime}, \sigma^{\prime}\right)$ we can change $L(v)$ into $L\left(v^{\prime}\right)=1$. Let $D$ be the Jacobian determinant $D=\frac{\partial\left(\tau^{\prime}, \sigma^{\prime}\right)}{\partial(\tau, \sigma)}>0$, then we have

$$
v^{\prime \mu \nu}:=\frac{\partial x^{\mu}}{\partial \tau^{\prime}} \frac{\partial x^{\nu}}{\partial \sigma^{\prime}}-\frac{\partial x^{v}}{\partial \tau^{\prime}} \frac{\partial x^{\mu}}{\partial \sigma^{\prime}}=\frac{1}{D} v^{\mu \nu}, \quad L\left(v^{\prime}\right)=\frac{1}{D} L(v)
$$

Thus, every reparametrization with $D=L(v)$ leads to $L\left(v^{\prime}\right)=1$. An example of such a reparametrization is given by

$$
\tau^{\prime}=\tau, \quad \sigma^{\prime}=\int_{0}^{\sigma} L(v(\tau, \hat{\sigma})) d \hat{\sigma}
$$

or by the parameter transformation (17).

As $s_{(\mu v)}=L_{(\mu v)}(v)=L_{(\mu v)}\left(v^{\prime}\right)$ on $\Sigma^{(2)}$, the field $s_{\mu v}(x)$ is not affected by a reparametrization. Thus, for the reparametrized $x\left(\tau^{\prime}, \sigma^{\prime}\right)$ with $L\left(v^{\prime}\right)=1$

$$
F_{\mu v}(x):=-\lambda s_{\mu \nu}(x)
$$

is a Maxwell field which satisfies the conditions (5) and (6).

Remark 2. The Maxwell field $F_{\mu \nu}$ is not uniquely determined by condition (3):

$$
F^{\mu v}(x(\tau, \sigma))=\lambda v^{\mu v}(\tau, \sigma) .
$$

Example. For the string motion (1) there exist at least two Maxwell fields of rank 2 , which satisfy condition (3) for $\frac{\pi}{2}<\sigma \leqq \pi$. The first one is the field (4) constructed by Kastrup, and a second one is the following:

$$
\begin{array}{ll}
F^{\prime 01}=\lambda A \frac{x^{1}}{\varrho}, & F^{\prime 02}=\lambda A \frac{x^{2}}{\varrho}, \quad F^{\prime 03}=0 \\
F^{\prime 12}=-\lambda A \omega \varrho, & F^{\prime 13}=F^{\prime 23}=0, \quad \varrho=\left[\left(x^{1}\right)^{2}+\left(x^{2}\right)^{2}\right]^{1 / 2} .
\end{array}
$$

This field $F^{\mu \nu}$ also fulfills condition (6) - in contrast to the field (4).

For the Proof of Theorem 2 we need the following theorem from [8].

Theorem B. Let the 2-dimensional surface $\Sigma^{(2)}$ with the twice continuously differentiable parametrization $x(\tau, \sigma)$ be imbedded in a geodesic field $(x, \Phi(x))$ with respect to $L$. Then $x(\tau, \sigma)$ is a solution of the equations of motion (9).

Now, we are prepared for the Proof of Theorem 2:

From assumption (7) we get $F^{\mu \nu} \neq 0$ and therefore $\lambda \neq 0$. We introduce the continuously differentiable functions

$$
\Phi^{\mu v}(x):=\frac{\lambda}{|\lambda|}\left[-\frac{1}{2} F_{\varrho \sigma} F^{\varrho \sigma}\right]^{-1 / 2} F^{\mu v}(x)
$$

with the following properties:

$$
\Phi^{\mu v}(x)=-\Phi^{v \mu}(x), \quad \Phi^{\mu \nu}(x) \varepsilon_{\mu \nu \varrho \sigma} \Phi^{\varrho \sigma}(x)=0 .
$$


Therefore, $\left(x, \Phi^{\mu \nu}(x)\right)=(x, \Phi(x))$ is a slope field of 2-dimensional surface elements. The differential form

$$
\Omega=L_{(\mu \nu)}(\Phi(x)) d x^{\mu} \wedge d x^{\nu}=-\Phi_{(\mu \nu)}(x) d x^{\mu} \wedge d x^{\nu}
$$

is closed, because

$$
\varepsilon_{\mu \nu \varrho \sigma} \partial^{\mu} \Phi^{\rho \sigma}(x)=0 .
$$

Thus, $(x, \Phi(x))$ is a geodesic field with respect to $L$. On the surface $\Sigma^{(2)}$ we have

$$
v^{\mu \nu}(\tau, \sigma)=\frac{1}{|\lambda|}\left[-\frac{1}{2} F_{\alpha \beta} F^{\alpha \beta}\right]^{1 / 2} \Phi^{\mu \nu}(x(\tau, \sigma)) .
$$

Hence, $\Sigma^{(2)}$ is embedded in the geodesic field $(x, \Phi(x))$. Using Theorem B we conclude that $x(\tau, \sigma)$ is a solution of the equations of motion (9).

We want to add a last remark: Among all the Maxwell fields which we studied in connection with a given string motion there is a preferred one: It is the field $s_{\mu \nu}(x)$ which is "normalized" by the Hamilton-Jacobi equation (15) and invariant under reparametrizations of the string. A further study of this field might be useful.

Acknowledgment. I am indebted to Prof. Kastrup for drawing my attention to this problem, for helpful discussions and a critical reading of the manuscript.

\section{References}

1. Kastrup, H.A.: Phys. Lett. 78B, 433 (1978)

2. Kastrup, H.A.: Phys. Lett. 82B, 237 (1979)

3. Rebbi, C.: Phys. Rep. 12C, 1 (1974)

4. Scherk, J.: Rev. Mod. Phys. 47, 123 (1975)

5. Carathéodory, C.: Acta Sci. Math. (Szeged) 4, 193 (1929). Reprinted in : Carathéodory, C.: Gesamm. Math. Schriften, Vol. 1, p. 401. München: Beck 1954

6. Velte, W.: Math. Z. 60, 367 (1954)

7. Velte, W.: Mitt. Math. Sem. No. 45, Giessen 1953

8. Klötzler, R.: Ber. Verh. Sächs. Akad. Wiss., Math.-nat. K1. 104, No. 6 (1961)

9. Godbillon, C.: Géométrie différentielle et méchanique analytique, Chap. VI. Paris: Hermann 1969

Communicated by R. Haag

Received October 1, 1979 
